\title{
ON THE CHOICE OF PARAMETER IN A METHOD FOR THE INVERSION OF FOURIER SERIES
}

\author{
M. DI NATALE, L. GOTUSSO, R. PAVANI, AND D. ROUX
}

\begin{abstract}
The a priori evaluation of the pointwise approximation, by a regularization method suggested earlier, of a $L^{1}$ periodic function $f$, when only noisy Fourier coefficients of $f$ are known, is given here in a formulation more suitable for numerical verification. A careful evaluation of the constants involved in the formulas is also provided. Finally, a procedure is described of a statistical check performed in order to verify whether the theoretically suggested value of the regularization parameter is a good choice with respect to functions generally arising in applications.
\end{abstract}

\section{INTRODUCTION}

We consider the (generally) ill-posed problem of "reconstructing" a function $f$, integrable on the $N$-dimensional torus, when we only know the sequence of its Fourier coefficients. A recent paper [2] (see also [1]) describes a regularization method to solve this problem which is stable also in the case of noisy data. Extensive numerical experience shows that the method is very efficient [4] (see also [5]). The efficiency is closely related to the choice of the regularization parameter $\sigma$. In [2], for large classes of functions, evaluations are given of the difference between $f$ and its approximation $\tilde{f}_{\sigma}$, in dependence on $\sigma$ and on the error of the Fourier coefficients. These evaluations, by a standard method, suggest a choice of a "good" value of $\sigma$. It is of interest to verify if this theoretical suggestion leads to a good choice of $\sigma$ for the functions generally arising in applications.

In this paper we give the results of a statistical check on the proposed value of $\sigma$ in the case of pointwise approximation. In $\S 2$ we briefly recall the regularization method and the results on pointwise approximation. In $\S 3$ we state the formulas which give the values of the constants involved in the estimation of $\sigma$. A careful evaluation of these constants is very important because (as we said) the efficiency of the method depends heavily on the choice of $\sigma$. In $\S 4$ we illustrate the procedure of our statistical check; in $\S 5$ we give, and comment on, some tables of the results obtained, and in $\S 6$ we briefly describe the algorithms and the program.

Received July 13, 1990; revised February 19, 1991.

1991 Mathematics Subject Classification. Primary 42A20.

This work was supported by Italian M.U.R.S.T. 


\section{THE REGULARIZATION METHOD}

We denote by $T^{N} \cong\left[-\frac{1}{2}, \frac{1}{2}\right)^{N}$ the $N$-dimensional torus and by $Z^{N}$ the lattice of integer points of $\mathbb{R}^{N}$. Let $f \in L^{1}\left(T^{N}\right)$ and $\hat{f}=\left\{\hat{f}_{n}\right\}_{n \in Z^{N}} \in l^{\infty}\left(Z^{N}\right)$ be the sequence of its Fourier coefficients. Let $P: \mathbb{R}^{N} \rightarrow \mathbb{R}$ be a homogeneous polynomial of even degree $k>0$ such that $P(x)>0$ if $x \neq 0$ and, for every $\lambda=\left\{\lambda_{n}\right\}_{n \in Z^{N}} \in l^{\infty}\left(Z^{N}\right)$ and $\sigma>0$, set (formally)

$$
R_{\sigma} \lambda \sim \sum_{n \in Z^{N}} \frac{\lambda_{n}}{1+\sigma P(n)} e^{2 \pi i n t}, \quad t \in T^{N} .
$$

If $k>N$, the right-hand side of (2.1) defines a continuous function of $t$.

Consider now a point $t$ where $f$ satisfies the condition

$$
\int_{|x-t| \leq r}|\bar{f}(x)-f(t)| d x \leq K r^{N+\alpha} \quad \forall r, 0<r \leq \frac{\sqrt{N}}{2}
$$

( $\bar{f}$ is the periodic continuation of $f$ in $\mathbb{R}^{N}$ ), for some $K, 0<K<+\infty$, and $\alpha, 0<\alpha \leq 1$. At such a point, if $k>N$, the following result holds [2, Theorem 6]: For every $\delta>0$ and $\sigma, 0<\sigma \leq 1$, we have

$$
\left|f(t)-R_{\sigma} \lambda(t)\right|<K c_{\alpha, N} \sigma^{\alpha / k}+a_{1, N} \sigma^{-N / k} \delta,
$$

where $\delta=\|\hat{f}-\lambda\|_{\infty}$ and the constants $c_{\alpha, N}$ and $a_{1, N}$ depend only on $P$.

Let us now set

$$
\delta_{0}=\left|\hat{f}_{0}-\lambda_{0}\right|, \quad \delta_{1}=\sup \left\{\left|\hat{f}_{n}-\lambda_{n}\right|: n \in Z^{N}, n \neq 0\right\} .
$$

An examination of the proof of (2.3) shows that the following more general result holds:

If (2.2) is satisfied and $k>N$, then for every $\sigma_{0}>0$ there exist $\bar{a}_{N}$ and $\bar{c}_{\alpha, N}=\bar{c}_{\alpha, N}\left(\sigma_{0}\right)$ such that

$$
\left|f(t)-R_{\sigma} \lambda(t)\right| \leq K \bar{c}_{\alpha, N} \sigma^{\alpha / k}+\bar{a}_{N} \sigma^{-N / k} \delta_{1}+\delta_{0}
$$

for every $\delta_{1}, \delta_{0} \geq 0$ and for every $\sigma, 0<\sigma \leq \sigma_{0}$.

By the minimum principle of majorant estimates applied to (2.4) (see, e.g., [7, pp. 18-19]) we obtain that, if

$$
\sigma=\bar{\sigma}=\left(\frac{N \bar{a}_{N} \delta_{1}}{\alpha K \bar{c}_{\alpha, N}}\right)^{k /(\alpha+N)},
$$

then

$$
\left|f(t)-R_{\sigma} \lambda(t)\right| \leq\left(1+\frac{N}{\alpha}\right)\left(\frac{\alpha}{N} K \bar{c}_{\alpha, N}\right)^{N /(\alpha+N)} \cdot\left(\bar{a}_{N} \delta_{1}\right)^{\alpha /(\alpha+N)}+\delta_{0} .
$$

The aim of the paper is to examine whether $\bar{\sigma}$ is a good choice of $\sigma$ for some typical test functions.

\section{Evaluation of $\bar{a}_{1}$ AND $\bar{c}_{1,1}$}

We consider the case $\alpha=1$ (the usual case), $N=1$, and we assume $P(x)=$ $x^{4}$ (so that $\left.k=4\right)$. In this case, $(2.5)$ and (2.6) become 


$$
\begin{gathered}
\sigma=\left(\frac{\bar{a}_{1} \delta_{1}}{K \bar{c}_{1,1}}\right)^{2}, \\
\left|f(t)-R_{\sigma} \lambda(t)\right| \leq 2\left(K \bar{c}_{1,1} \bar{a}_{1} \delta_{1}\right)^{1 / 2}+\delta_{0} .
\end{gathered}
$$

The procedure of evaluating $\bar{a}_{1}$ and $\bar{c}_{1,1}$ starts as in [2, Theorems 4 and 5], respectively. Obviously,

$$
\left|f(t)-R_{\sigma} \lambda(t)\right| \leq\left|f(t)-R_{\sigma} \hat{f}(t)\right|+\left|R_{\sigma}(\hat{f}-\lambda)(t)\right| .
$$

We have

$$
\left|R_{\sigma}(\hat{f}-\lambda)(t)\right| \leq \delta_{0}+\left|\sum_{\substack{-\infty \\ n \neq 0}}^{\infty} \frac{\hat{f}_{n}-\lambda_{n}}{1+\sigma n^{4}} e^{2 \pi i n t}\right| \leq \delta_{0}+2 \delta_{1} \sum_{n=1}^{\infty}\left(1+\sigma n^{4}\right)^{-1}
$$

Since

$$
\sum_{n=1}^{\infty} \frac{1}{1+\sigma n^{4}}<\int_{0}^{+\infty} \frac{d x}{1+\sigma x^{4}}=\sigma^{-1 / 4} \int_{0}^{+\infty} \frac{d y}{1+y^{4}}=\frac{\pi}{2 \sqrt{2}} \sigma^{-1 / 4}
$$

we obtain

$$
\bar{a}_{1}<\frac{\pi}{\sqrt{2}}=2.2214414 \ldots
$$

Consider now the function $G: \mathbb{R} \rightarrow \mathbb{R}$,

$$
G(x)=\pi e^{-\sqrt{2} \pi|x|} \sin \left(\frac{\pi}{4}+\sqrt{2} \pi|x|\right),
$$

whose Fourier transform is $\widehat{G}(x)=\left(1+x^{4}\right)^{-1}[3$, p. 9, formula (19)]. If we set

$$
G_{\sigma}(x)=\sigma^{-1 / 4} G\left(\sigma^{-1 / 4} x\right),
$$

the series $\sum_{n=-\infty}^{\infty} G_{\sigma}(x+n)$ defines a periodic function $K_{\sigma} \in L^{1}(T)$ whose Fourier series is [9, p. 260, Theorem 3.8]

$$
K_{\sigma}(t)=\sum_{n=-\infty}^{\infty} \frac{1}{1+\sigma n^{4}} e^{2 \pi i n t}
$$

This allows us to evaluate $\left|f(t)-R_{\sigma} \hat{f}(t)\right|$. We can always assume $t=0$. Since $\int_{T} K_{\sigma}(x) d x=1$, we have

$$
f(0)=\int_{T} f(0) K_{\sigma}(x) d x
$$

Since

$$
R_{\sigma} \hat{f}(0)=K_{\sigma} * f(0)=\int_{T} K_{\sigma}(x) f(-x) d x
$$

we obtain

$$
\begin{aligned}
R_{\sigma} \hat{f}(0)-f(0) & =\int_{T}(f(-x)-f(0)) K_{\sigma}(x) d x \\
& =\sum_{n=-\infty}^{\infty} \int_{T}(f(-x)-f(0)) G_{\sigma}(x+n) d x
\end{aligned}
$$

For the sake of simplicity, we set

$$
|f(x)-f(0)|=\Delta f(x), \quad 2^{1 / 2} \pi \sigma^{-1 / 4}=c .
$$


Then

$$
\begin{aligned}
\left|R_{\sigma} \hat{f}(0)-f(0)\right| & \leq \pi \sigma^{-1 / 4} \sum_{n=-\infty}^{\infty} \int_{T} \Delta f(-x) e^{-c|x+n|} d x \\
& =\pi \sigma^{-1 / 4} \sum_{n=-\infty}^{\infty} I_{n}
\end{aligned}
$$

We have

$$
\begin{aligned}
\sum_{n \neq 0} I_{n} & =\sum_{n=1}^{\infty} \int_{-1 / 2}^{1 / 2} \Delta f(-x)\left(e^{-c(x+n)}+e^{-c(-x+n)}\right) d x \\
& =\sum_{n=1}^{\infty} e^{-c n}\left\{\int_{-1 / 2}^{1 / 2} \Delta f(-x) e^{-c|x|} d x+\int_{-1 / 2}^{1 / 2} \Delta f(-x) e^{c|x|} d x\right\} \\
& =\sum_{n=1}^{\infty} e^{-c n}\left\{I_{0}+I^{*}\right\}
\end{aligned}
$$

From (2.2) we obtain

$$
I^{*} \leq e^{c / 2} \int_{-1 / 2}^{1 / 2} \Delta f(x) d x \leq K 2^{-2} e^{c / 2} .
$$

Moreover,

$$
\begin{aligned}
I_{0} & =\int_{0}^{1 / 2}\{\Delta f(-x)+\Delta f(x)\} e^{-c x} d x \\
& =\left.e^{-c x} \int_{-x}^{x} \Delta f(t) d t\right|_{0} ^{1 / 2}+c \int_{0}^{1 / 2} \int_{-x}^{x} \Delta f(t) d t e^{-c x} d x \\
& \leq K e^{-c / 2} \cdot 2^{-2}+K c \int_{0}^{1 / 2} x^{2} e^{-c x} d x \\
& =\frac{2 K}{c^{2}}\left\{1-e^{-c / 2}\left(\frac{c}{2}+1\right)\right\}
\end{aligned}
$$

Since $e^{-x}(x+1)<1$ for all $x>0$, we have

$$
I_{0} \leq \frac{2 K}{c^{2}} \quad \forall \sigma>0
$$

From (3.3), (3.4), (3.5), (3.6) we obtain for every $\sigma>0$

$$
\begin{aligned}
\left|R_{\sigma} \hat{f}(0)-f(0)\right| & \leq\left(\sum_{n=0}^{\infty} e^{-c n} I_{0}+\sum_{n=1}^{\infty} e^{-c n} I^{*}\right) \pi \sigma^{-1 / 4} \\
& \leq \frac{\pi \sigma^{-1 / 4}}{1-e^{-c}}\left\{\frac{2 K}{c^{2}}+\frac{K}{4} e^{-c / 2}\right\} \\
& =\frac{K \sigma^{1 / 4}}{1-\exp \left(-2^{1 / 2} \pi \sigma^{-1 / 4}\right)}\left\{\frac{1}{\pi}+\frac{\pi}{4} \sigma^{-1 / 2} \exp \left(-2^{-1 / 2} \pi \sigma^{-1 / 4}\right)\right\}
\end{aligned}
$$

Since the function $\varphi(y)=y^{2} \exp \left(-2^{-1 / 2} \pi y\right)$ attains its maximum at $y=$ $2^{3 / 2} / \pi,(2.4)$ holds. In particular, if $0<\sigma_{0} \leq \pi^{4} / 2^{6}$, we can assume 


$$
\bar{c}_{1,1}=\bar{c}_{1,1}\left(\sigma_{0}\right)=\frac{1}{1-\exp \left(-2^{1 / 2} \pi \sigma_{0}^{-1 / 4}\right)}\left\{\frac{1}{\pi}+\frac{\pi}{4} \sigma_{0}^{-1 / 2} \exp \left(-2^{-1 / 2} \pi \sigma_{0}^{-1 / 4}\right)\right\} \text {. }
$$

If $\sigma_{0}=0.25$, we have

$$
\bar{c}_{1,1}(0.25) \leq\left(1-e^{-2 \pi}\right)^{-1}\left(\frac{1}{\pi}+\frac{\pi}{2} e^{-\pi}\right) \cong 0.3869
$$

\section{THE STATISTICAL CHECK}

A test is now described which has the aim of examining whether the value $\bar{\sigma}$ of $\sigma$ given by (3.1) is in every case a better choice than $\sigma=0$ and in general is a good choice for the regularization parameter. We are also interested in verifying that $\bar{\sigma}$ is a good starting point for an iterative process which allows us to obtain a very good value of $\sigma$ quickly for the particular pairs $(f, t)$ considered.

The statistical check involves the following test functions:

$$
\begin{array}{lll}
f_{1}(t)=\operatorname{sgn} t, & f_{2}(t)=t, & f_{3}(t)=t^{2}, \\
f_{4}(t)=t^{3}, & f_{5}(t)=e^{t}, & f_{6}(t)=\left(1-4 t^{2}\right)^{-1 / 2} .
\end{array}
$$

For these functions we know the values of $K=K(t)$ for every $t \in T$ (see [6]).

In our test we consider the values $K=0.45,1,4,22$ because these values are assumed by $K\left(t ; f_{j}\right)$ for several functions $f_{j}(j=1, \ldots, 6)$ and $t \in T$. Moreover, these points are in different positions with respect to the "critical set" of $f_{j}$ (i.e., the set of points where $f_{j}$ or its derivatives are discontinuous).

We always take $\delta_{0}=0$ (i.e., $\lambda_{0}=\hat{f}_{0}$ ). For $n>0$ we take $\lambda_{-n}=\bar{\lambda}_{n}$ (since the test functions are real-valued) and $\lambda_{n}=\hat{f}_{n}+\eta v_{n}$, where $\eta$ is a proper constant and $\left\{v_{n}\right\}$ is a pseudorandom sequence with normal distribution, zero mean, and unit variance.

We approximate the test functions by the Fourier polynomials of $R_{\sigma} \lambda$,

$$
P_{m, \sigma}=\sum_{n=-m}^{m} \frac{\lambda_{n}}{1+\sigma n^{4}} e^{2 \pi i n t}
$$

This truncation of $R_{\sigma} \lambda$ is equivalent to a further perturbation of the data $\left(\lambda_{n}=0\right.$ if $\left.|n|>m\right)$.

In our experiments we set $m=20$ or $m=50$. Since for all our test functions the modulus of the coefficients $\hat{f}_{n} \neq 0$ decreases with $|n|$ for sufficiently large $n$, the error $\varepsilon_{1}$ on $\hat{f}$ due to truncation (in $l^{\infty}$ ) is $\varepsilon_{1}=\left|\hat{f}_{m+1}\right|$ and, if we set $\varepsilon_{2}=\max \left\{\left|\lambda_{n}-\hat{f}_{n}\right|: 0<n \leq m\right\}$, we have

$$
\delta=\delta_{1}=\max \left(\varepsilon_{1}, \varepsilon_{2}\right) \text {. }
$$

For every pseudorandom sequence $\left\{v_{n}\right\}$ considered, and for every $f_{j}$ and $m$, we choose $\eta$ in order to have $\delta=0.025$.

Conjecturing (as one always does in the experiments) the values of the parameter $\sigma$ involved in our check to be smaller than 0.25 , we assume $\bar{c}_{1,1}=$ $\bar{c}_{1,1}(0.25)$ and we calculate $\bar{\sigma}$ (defined in (3.1)) and the right-hand side $M(K, \delta)$ of (3.2). For every pair $\left(f_{j}, t\right)$, by using the routine described in $\S 6$, we calculate the value $\sigma^{*}=\sigma^{*}(\lambda)$ nearest to $\bar{\sigma}$ which minimizes $\Delta_{\sigma}=$ $\left|f_{j}(t)-P_{m, \sigma}(t)\right|$ and

$$
\Delta_{\bar{\sigma}}=\left|f_{j}(t)-P_{m, \bar{\sigma}}(t)\right|, \quad \Delta_{\sigma^{*}}=\left|f_{j}(t)-P_{m, \sigma^{*}}(t)\right|, \quad \Delta_{0}=\left|f_{j}(t)-P_{m, 0}(t)\right| .
$$


We iterate the process with the same value $\delta=0.025$ for 100 different sequences $\left\{v_{n}\right\}$. Then we calculate the mean values $\bar{\sigma}^{*}, \bar{\Delta}_{\bar{\sigma}}, \bar{\Delta}_{\sigma^{*}}, \bar{\Delta}_{0}$, and their variances. Subsequently, we choose other values of $\delta$ and we perform for these $\delta$ the same operations of the case $\delta=0.025$. We proceed as described for the values $K=0.45,1,4,22$.

\section{TABLE AND COMMENTS}

For the sake of brevity, we confine ourselves to presenting some global results for the case $K=4$ and $m=50$. The other results obtained are similar and are available from the second author (L.G.). Obviously, also for $K=4$, we cannot present tables which for every choice of the data $f, t, \delta$ contain the values

$$
K=4
$$

\begin{tabular}{|c|c|c|c|c|c|c|c|}
\hline$f$ & $t$ & $\delta$ & $\bar{\sigma}$ & $\overline{\sigma^{*}}$ & $\bar{\Delta}_{\bar{\sigma}}$ & $\bar{\Delta}_{\sigma^{*}}$ & $\overline{\Delta_{0}}$ \\
\hline \multirow[t]{12}{*}{1} & \multirow[t]{3}{*}{$1.2 \mathrm{E}-1$} & $2.5 E-2$ & $1.3 E-3$ & $7.3 E-3$ & $5.3 E-2$ & $3.1 E-3$ & $8.1 E-2$ \\
\hline & & $3.7 E-2$ & $2.9 E-3$ & $1.1 \mathrm{E}-2$ & $6.9 E-2$ & $2.7 E-3$ & $1.2 \mathrm{E}-1$ \\
\hline & & $1.2 \mathrm{E}-2$ & $3.2 E-4$ & $2.7 E-4$ & $1.5 \mathrm{E}-2$ & $3.7 E-3$ & $4.3 E-2$ \\
\hline & \multirow[t]{3}{*}{$1.3 \mathrm{E}-1$} & $2.5 E-2$ & $1.3 E-3$ & $7.6 \mathrm{E}-3$ & $4.8 E-2$ & $2.8 E-3$ & $8.4 E-2$ \\
\hline & & $3.7 E-2$ & $2.9 \mathrm{E}-3$ & $1.5 \mathrm{E}-2$ & $6.8 E-2$ & $2.4 \mathrm{E}-3$ & $1.2 \mathrm{E}-1$ \\
\hline & & $1.2 \mathrm{E}-2$ & $3.2 \mathrm{E}-4$ & $3.9 E-4$ & $1.3 \mathrm{E}-2$ & $3.5 \mathrm{E}-3$ & $4.1 E-2$ \\
\hline & \multirow[t]{3}{*}{$1.7 \mathrm{E}-1$} & $2.5 E-2$ & $1.3 \mathrm{E}-3$ & $1.3 E-3$ & $2.6 \mathrm{E}-2$ & $1.0 \mathrm{E}-2$ & $9.3 E-2$ \\
\hline & & $3.7 \mathrm{E}-2$ & $2.9 \mathrm{E}-3$ & $2.1 \mathrm{E}-3$ & $4.2 \mathrm{E}-2$ & $1.5 \mathrm{E}-2$ & $1.2 \mathrm{E}-1$ \\
\hline & & $1.2 \mathrm{E}-2$ & $3.2 E-4$ & $5.8 E-4$ & $1.3 E-2$ & $3.5 E-3$ & $3.2 \mathrm{E}-2$ \\
\hline & \multirow[t]{3}{*}{$2.5 \mathrm{E}-1$} & $2.5 \mathrm{E}-2$ & $1.3 \mathrm{E}-3$ & $3.3 E-3$ & $2.3 E-2$ & $5.8 \mathrm{E}-3$ & $9.2 \mathrm{E}-2$ \\
\hline & & $3.7 \mathrm{E}-2$ & $2.9 \mathrm{E}-3$ & $3.7 \mathrm{E}-3$ & $3.4 \mathrm{E}-2$ & $1.1 \mathrm{E}-2$ & $1.3 \mathrm{E}-1$ \\
\hline & & $1.2 \mathrm{E}-2$ & $3.2 E-4$ & $1.3 \mathrm{E}-3$ & $1.4 \mathrm{E}-2$ & $4.7 E-3$ & 4.1E - 2 \\
\hline \multirow[t]{3}{*}{4} & \multirow[t]{3}{*}{$4.7 \mathrm{E}-1$} & $2.5 \mathrm{E}-2$ & $1.3 \mathrm{E}-3$ & $1.9 \mathrm{E}-3$ & $5.4 \mathrm{E}-2$ & $1.3 \mathrm{E}-2$ & $7.7 \mathrm{E}-2$ \\
\hline & & $2.3 E-3$ & $1.1 E-5$ & $3.5 E-5$ & $8.8 \mathrm{E}-3$ & $2.0 \mathrm{E}-4$ & $1.1 \mathrm{E}-2$ \\
\hline & & $7.8 E-4$ & $1.2 \mathrm{E}-6$ & $6.2 \mathrm{E}-7$ & $3.2 \mathrm{E}-3$ & $1.9 \mathrm{E}-3$ & $7.9 E-3$ \\
\hline \multirow[t]{6}{*}{5} & \multirow[t]{3}{*}{$-4.4 \mathrm{E}-1$} & $2.5 \mathrm{E}-2$ & $1.3 \mathrm{E}-3$ & $6.9 E-4$ & $3.9 \mathrm{E}-2$ & $3.3 E-3$ & $1.0 \mathrm{E}-1$ \\
\hline & & $9.8 \mathrm{E}-3$ & $2.0 \mathrm{E}-4$ & $4.3 E-4$ & $3.1 E-2$ & $9.9 E-4$ & $3.6 \mathrm{E}-2$ \\
\hline & & $3.2 \mathrm{E}-3$ & $2.2 \mathrm{E}-5$ & $7.7 \mathrm{E}-6$ & $1.4 \mathrm{E}-2$ & $2.4 \mathrm{E}-3$ & $1.3 \mathrm{E}-2$ \\
\hline & \multirow[t]{3}{*}{$4.3 \mathrm{E}-1$} & $2.5 \mathrm{E}-2$ & $1.3 \mathrm{E}-3$ & $1.2 \mathrm{E}-3$ & $2.8 \mathrm{E}-2$ & $5.0 \mathrm{E}-3$ & $9.9 \mathrm{E}-2$ \\
\hline & & $9.7 E-3$ & $2.0 \mathrm{E}-4$ & $6.9 E-4$ & $3.4 \mathrm{E}-2$ & $1.5 E-3$ & $3.9 \mathrm{E}-2$ \\
\hline & & $3.2 \mathrm{E}-3$ & $2.2 \mathrm{E}-5$ & $1.5 \mathrm{E}-5$ & $6.9 E-3$ & $2.4 \mathrm{E}-3$ & $1.2 \mathrm{E}-2$ \\
\hline \multirow[t]{3}{*}{6} & \multirow[t]{3}{*}{$2.1 \mathrm{E}-1$} & $7.0 \mathrm{E}-2$ & $1.0 \mathrm{E}-2$ & $4.2 \mathrm{E}-2$ & $4.6 \mathrm{E}-2$ & $8.1 E-4$ & $1.1 \mathrm{E}-1$ \\
\hline & & $2.1 \mathrm{E}-1$ & $9.1 \mathrm{E}-2$ & $6.4 \mathrm{E}-2$ & $7.2 \mathrm{E}-2$ & $2.1 \mathrm{E}-2$ & $6.1 \mathrm{E}-1$ \\
\hline & & $7.0 \mathrm{E}-2$ & $1.0 \mathrm{E}-2$ & $4.6 \mathrm{E}-2$ & $6.7 E-2$ & $4.6 \mathrm{E}-3$ & $2.4 \mathrm{E}-1$ \\
\hline
\end{tabular}


$\sigma^{*}, \Delta_{\bar{\sigma}}, \Delta_{\sigma^{*}}$, and $\Delta_{0}$ of all the hundred performed experiments; we therefore present only their means. On the other hand, the variances are very small; so our choice is a posteriori justified.

First of all, let us compare $\bar{\Delta}_{\bar{\sigma}}$ and $\bar{\Delta}_{0}$. In general, for every $K$ we have

$$
\bar{\Delta}_{\bar{\sigma}}<\bar{\Delta}_{0}
$$

with the exception of $K=22$ for some values of $t$; a careful examination of the results shows that this anomaly is essentially due to the fact that the points $t$ considered are close to the "critical set" of $f_{j}$ (see $\S 4$ ), and $\sigma^{*}$ is close to zero. We remark, however, that also when $K=22,(5.1)$ holds for every $t$ if $\delta$ is sufficiently small. Finally, we note that in all the experiments with noisy data we have

$$
\bar{\Delta}_{\sigma^{*}} \ll \bar{\Delta}_{0} .
$$

The result of our check is the following. The choice of $\bar{\sigma}$ does not seem to be satisfactory in order to obtain a severe reduction of $\Delta_{\sigma}$ with respect to $\Delta_{0}$; nevertheless, (5.1) holds when $t$ is sufficiently far from the critical set. Moreover, the results show that $\bar{\sigma}$ is a good estimate of the order of magnitude of $\sigma^{*}$. This reveals that $\Delta_{\sigma}$ is generally very sensitive to small variations of $\sigma$ and shows that $\bar{\sigma}$ is a good starting value for an iterative process converging to a very good value of $\sigma$, that is, $\sigma^{*}$.

\section{AlgorithMS AND PROGRAM}

In order to carry out the computations described above, we implemented a code in FORTRAN 77 using structured programming techniques. It carries out the following tasks:

(1) it reads input data;

(2) it computes the Fourier coefficients;

(3) it executes the following loop as many times as needed (each time with a different random deviate):

(a) generate a set of random numbers with a normal distribution (with zero mean and unit variance);

(b) determine the random number with the greatest absolute value;

(c) compute the noisy Fourier coefficients using the generated random numbers $v_{n}$ multiplied by $\eta=\varepsilon_{2} / \max \left(\left|v_{n}\right|, n=1, \ldots, m\right)$, where $\varepsilon_{2}$ depends on $\delta$ as explained in $\S 4$;

(d) find the best smoothing coefficient (called $\sigma^{*}$ ) by means of the minimization of $\Delta_{\sigma}$

(e) compute and print the following values: $\delta, \varepsilon_{1}, \varepsilon_{2}, \bar{\sigma}, M(k, \delta), \bar{\sigma}^{*}$, $\operatorname{var} \sigma^{*}, \bar{\Delta}_{\bar{\sigma}}, \operatorname{var} \Delta_{\bar{\sigma}}, \bar{\Delta}_{\sigma^{*}}, \operatorname{var} \Delta_{\sigma^{*}}, \bar{\Delta}_{0}, \operatorname{var} \Delta_{0}$.

Let us explain some of these tasks in more details:

(2) The Fourier coefficients of the functions $f_{i}(i=1, \ldots, 5)$ are provided by the formulas given in [4]; the integrals involved in the evaluation of the Fourier coefficients of function $f_{6}$ are computed as described in [4].

(3a) The kernel of the random number generator is the function RAN2 from [8] that returns a uniform random deviate between 0 and 1 , with any negative number as a seed of the sequence; from two different uniform deviates on $(0,1)$ we obtained a normally distributed deviate $v_{n}$ with zero mean and unit variance using the Box-Muller transformation. 
(3b) In order to sort an array of length $N$ in ascending order, we used the Heapsort Algorithm as implemented in the subroutine SORT from [8]; it is an $\left(\mathrm{N} \log _{2} N\right)$-order process, not only on average, but also for the worst-case input data.

(3d) The minimization process is carried out by Brent's method as implemented in the subroutine BRENT from [8]; starting from two distinct initial points, it performs an unconstrained minimization using parabolic interpolation and returns the minimum function value with an assigned precision. Computing $\sigma^{*}$ is in reality a constrained minimization problem subject to $\sigma \geq 0$; we overcome this difficulty by introducing a new variable $\sigma=\varphi^{2}$ and computing the unconstrained minimum of $\Delta_{\varphi^{2}}=\left|f(t)-P_{m, \varphi^{2}}(t)\right|$ with initial points $\varphi_{1}=0.0 ; \varphi_{2}=\sqrt{1.2 \bar{\sigma}}$.

Our computations were carried out on a Micro-VAX II computer, with a memory requirement of about eight Kbyte.

\section{BIBLIOGRAPHY}

1. Abstracts Amer. Math. Soc. 10 (1989), p. 235.

2. L. De Michele, M. Di Natale, and D. Roux, Sharp properties of a regularization method for inversion of Fourier series, Proc. Meeting "Trends in Functional Analysis and Approximation Theory," Atti Sem. Mat. Fis. Univ. Modena 39 (1991), 253-267.

3. A. Erdélyi, W. Magnus, F. Oberhettinger, and F. G. Tricomi, Tables of integral transforms, Vol. 1, McGraw-Hill, New York, 1954.

4. L. Gotusso, R. Pavani, and D. Roux, Numerical results from a regularization method for inversion of Fourier series, Rend. Mat. (7) 10 (1990), 423-438.

5. L. Gotusso and D. Roux, Teoria, analisi numerica e valutazione dei risultati di un metodo per l'inversione delle serie di Fourier, Atti Accad. Sci. Lettere Arti Palermo (to appear).

6. L. Gotusso, D. Roux, and P. Zanzi, Sul calcolo di una costante relativa ad un metodo di inversione delle serie di Fourier, Istit. Lombardo Accad. Sci. Lett. Rend. A 124 (1990), 85-98.

7. V. A. Morozov, Methods for solving incorrectly posed problems, Springer-Verlag, New York, 1984.

8. W. H. Press, B. P. Flannery, S. A. Teukolsky, and W. T. Vetterling, Numerical recipes, Cambridge Univ. Press, 1986.

9. E. M. Stein and G. Weiss, Introduction to Fourier analysis on Euclidean spaces, Princeton Univ. Press, Princeton, NJ, 1971.

(M. Di Natale and D. Roux) Dipartimento di Matematica, Università di Milano, via C. Saldini, 50, 20133 Milano, Italia

(L. Gotusso and R. Pavani) Dipartimento di Matematica, Politecnico di Milano, Piazza LEONARDo DA VincI, 32, 20131 Milano, Italia

E-mail address, L. Gotusso: laugot@ipmma1.polimi.it 